\title{
Discrete-Time Control of Continuous Systems with Approximate Decentralized Fixed Modes *
}

\author{
Amir G. Aghdam ${ }^{\text {a }}$, Edward J. Davison ${ }^{\mathrm{b}}$ \\ ${ }^{a}$ Department of Electrical and Computer Engineering, Concordia University, Montréal, Québec H3G 1 M8 Canada \\ ${ }^{\mathrm{b}}$ Department of Electrical and Computer Engineering, University of Toronto, Toronto, Ontario M5S 3G4 Canada
}

\begin{abstract}
In this paper, the discrete-time control of decentralized continuous-time systems, which have approximate decentralized fixed modes, is studied. It is shown that under certain conditions, discrete-time controllers can improve the overall performance of the decentralized control system, when a linear time-invariant continuous-time controller is ineffective. In order to obtain these conditions, a quantitative measure for different types of approximate fixed modes in a decentralized system is given. In this case, it is shown that discrete-time zero-order hold $(\mathrm{ZOH})$ controllers, and in particular, that generalized sampled-data hold functions (GSHF), can significantly improve the overall performance of the resultant closed-loop system. The proposed sampled-data controller is, in fact, a linear time-varying controller for the continuous-time system.
\end{abstract}

Key words: Decentralized control, Sampled-data control, Decentralized fixed modes, Approximate fixed modes.

\section{Introduction}

Decentralized fixed modes (DFM) were introduced in (Wang and Davison 1973), where it was shown that they played an essential role in determining if a LTI plant can be stabilized by applying a decentralized LTI controller. In particular, it was shown that a LTI plant can be stabilized using a decentralized LTI controller if and only if the plant has no unstable DFMs. The above paper also considered the case of more general information flow constraints other than decentralized, and in (Davison and Chang 1990), the above results were extended to the case of general proper systems. A graph-theoretic method was presented in (Lavaei and Aghdam 2007a) to find the unrepeated DFMs of LTI systems.

Several classifications have been introduced for DFMs. The notion of quotient fixed modes (QFM), introduced in (Gong and Aldeen 1997), refers to the fixed modes

\footnotetext{
ऋ This work has been supported in part by the Natural Sciences and Engineering Research Council of Canada under grant RGPIN-262127-03 and in part by the Natural Sciences and Engineering Research Council of Canada under grant No. A4396. Corresponding author A. G. Aghdam. Tel. (514)848-2424/4137. Fax (514)848-2802.
}

Email addresses: aghdam@ece.concordia.ca (Amir G. Aghdam), ted@control.utoronto.ca (Edward J. Davison). of the "quotient system" associated with strongly connected subsystems of the plant. They showed that QFMs remain "fixed" with respect to any type of decentralized output feedback, e.g., nonlinear or time-varying control. Thus, systems with unstable QFMs are not stabilizable with respect to any type of nonlinear or time-varying decentralized controller.

The notion of structurally fixed modes was introduced in (Sezer and Šiljak 1981), using the definition of a structured matrix and structurally equivalent matrices. This notion was used to identify the modes that cannot be shifted by decentralized feedback regardless of the numerical values of the system's nonzero parameters.

Structured DFMs introduced in (Özgüner and Davison 1985) are those modes (if any) of a decoupled statespace model $(C, A, B)$, that continue to be DFMs after perturbing the nonzero values of the system matrices $C, B$. It is well known that under certain conditions, discrete-time control can be used to stabilize an unstable continuous-time system which has unstable unstructured DFM present, e.g. see (Anderson and Moore 1981), (Özgüner and Davison 1985), (Khargonekar and Özgüler 1994). It was shown in (Özgüner and Davison 1985) that for almost all sampling periods, a sampled system has the same number of structured DFMs as the orig- 
inal continuous-time system, and has no unstructured DFMs, provided all unstructured DFMs are distinct and are nonzero eigenvalues of the system. It immediately follows that a continuous-time LTI system with unstable DFMs which are all unstructured (but distinct and nonzero), can be stabilized by using a discrete-time controller with a simple structure, e.g., with a zero-order hold $(\mathrm{ZOH})$. In this case, when the discrete-time equivalent model of the continuous-time system is obtained using a fixed sampling period, unstructured DFMs will generically no longer exist in the resultant discrete-time model, and an appropriate technique can then be used to design a discrete-time controller to stabilize the system. The overall controller then obtained consists of the above decentralized LTI controller and a ZOH. This configuration is equivalent to a time-varying continuous-time controller.

The idea of using generalized sampled-data hold functions (GSHF) instead of a simple $\mathrm{ZOH}$ (or firstorder hold) in control systems was first introduced in (Chammas and Leondes 1978). Kabamba examined the application of GSHFs in control systems, and pointed out that by using GSHF, one can obtain much of the efficiency of state feedback, without the requirement of state estimation (Kabamba 1987). He also showed that GSHFs can significantly improve the performance of the closed-loop system. Application of GSHFs in decentralized systems was also investigated in (Aghdam and Davison 1999), where it was shown that a discretetime decentralized controller with GSHF can result in a significant improvement compared to a simple $\mathrm{ZOH}$.

The application of GSHFs to decentralized control structure modification was in addition studied in (Aghdam et al. 2006). It was shown that under some conditions on the system's controllable and unobservable subspaces, GSHFs can be used to remove certain interconnections in the discrete-time equivalent model, so that the resultant digraph of the discrete-time model has a desirable acyclic form. As a result, one can apply centralized control design techniques to each subsystem independently to obtain a set of local controllers for the system in the discrete-time domain. In this case, the stability of each individual subsystem will guarantee the stability of the overall system (due to the acyclic structure of the discrete-time model) but depending on the strength of the interconnections between the subsystems, the overall performance may be poor. It is to be noted that a disadvantage of generalized sampled-data hold functions is that they are prone to robustness difficulties in the continuous time domain, e.g. see (Feuer and Goodwin 1994).

A multirate discrete-time decentralized control approach was used in (Scattolini and Schiavoni 1996) to stabilize a system, where the outputs of different subsystems are measured using different rates, but with the inputs simultaneously updated. It was also pointed out in (Scattolini and Schiavoni 1996) that the development of specific design procedures for decentralized stabilization is not a trivial problem. Design of GSHFs with a prespecified form (e.g., polynomial, piecewise constant, etc.), on the other hand, is investigated in (Lavaei and Aghdam 2007c) and a necessary and sufficient condition for the existence of a stabilizing GSHF of the desired form is derived. However, the problem of finding a stabilizing discrete-time decentralized controller (in the form of multirate control, GSHF, etc.) is in general not straightforward, especially when the system is "close" to having a fixed mode due to the close matching of parameters in the system (this will be clarified in Example 1).

This paper considers the case when the system has so-called unstructured approximate decentralized fixed modes (ADFM) (Vaz and Davison 1989), which implies that the system has a mode which is "close" to being an unstructured DFM. In this case, it will be shown that the application of discrete-time control using $\mathrm{ZOH}$ or generalized sampled-data functions can eliminate such ADFM, which in turn can thence improve the performance of the closed-loop system; as a result, decentralized digital controllers can potentially improve the overall performance of the closed-loop system, in contrast to the case when the system is controlled by a LTI continuous-time decentralized controller.

The organization of this paper is as follows: Section 2 presents the mathematical framework to formulate the effect of sampling on DFMs and ADFMs. Simulation results are given in Section 3, and finally Section 4 draws the concluding remarks.

\section{Sampling and Decentralized Control}

\subsection{Decentralized and Quotient Fixed Modes}

Brief descriptions of decentralized and quotient fixed modes are presented here. Consider the following system consisting of $m$ control agents:

$$
\begin{gathered}
\dot{x}(t)=A x(t)+\sum_{j=1}^{m} b_{j} u_{j}(t), \\
y_{j}(t)=c_{j} x(t), \quad j=1, \ldots, m
\end{gathered}
$$

where $x(t) \in \mathbb{R}^{n}$ is the state vector, and $u_{j}(t) \in \mathbb{R}^{q_{j}}$, and $y_{j}(t) \in \mathbb{R}^{m_{j}}, j \in \overline{\mathrm{m}}:=\{1, \ldots, m\}$ are the control vector and output vector of agent $j$ respectively, with $u:=\left[\begin{array}{lll}u_{1}^{\prime} & \ldots & u_{m}^{\prime}\end{array}\right]^{\prime}$ and $y:=\left[\begin{array}{lll}y_{1}^{\prime} & \ldots & y_{m}^{\prime}\end{array}\right]^{\prime}$. Furthermore, $A, b_{j}$, and $c_{j}$ are matrices of appropriate dimensions.

Definition 1 The decentralized fixed modes (DFM) of (1) are those modes of the system that remain fixed when LTI controllers with a diagonal information flow structure are applied (Wang and Davison 1973). In other 
words, $\lambda \in \mathbb{C}$ is a DFM of the system (1) with respect to a diagonal information flow matrix, if:

$$
\lambda \in \bigcap \operatorname{sp}\left(A+b_{1} K_{1} c_{1}+\ldots+b_{m} K_{m} c_{m}\right)
$$

for all gain matrices $K_{j}$, whose dimension is equal to the input-output size of control agent $j$, for all $j \in \overline{\mathrm{m}}$. Various techniques for the characterization of decentralized fixed modes are developed in (Davison and Chang 1990).

Corresponding to the decomposition of a system into strongly connected subsystems, one can define a new decentralized system with a control agent assigned to each subsystem, which is in fact the quotient system for (1) (Kobayashi and Yoshikawa 1982). As an illustration of this definition, consider a decentralized control system with the digraph of Figure 1. This system consists of three subsystems, and only two of these subsystems (subsystems 1 and 2) are strongly connected. The corresponding quotient system is depicted in Figure 2.

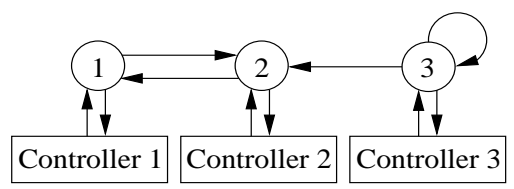

Fig. 1. A decentralized control system

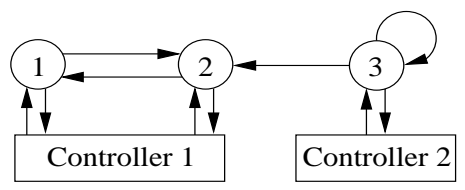

Fig. 2. A quotient system

Definition 2 Given the system (1), the DFMs of the so-called quotient system for (1) are called quotient fixed modes (QFM) of the system (1) (Gong and Aldeen 1997).

It was shown in (Gong and Aldeen 1997) that QFMs remain "fixed" with respect to any type of decentralized output feedback, e.g., nonlinear or time-varying control. It is desired now to show that for a LTI system with distinct and nonzero eigenvalues, the notions of QFM and structured DFM are equivalent.

\subsection{Effect of Sampling on DFMs}

It is known that sampling can eliminate distinct and nonzero unstructured DFMs (Özgüner and Davison 1985). The process of sampling the system (1) will now be investigated. The discrete-time equivalent model, corresponding to (1), is represented by:

$$
\begin{array}{r}
x[k+1]=A_{d} x[k]+\sum_{j=1}^{m} b_{d_{j}} u_{j}[k], \\
y_{j}[k]=c_{d_{j}} x[k], \quad j=1, \ldots, m
\end{array}
$$

where the discrete argument corresponding to the samples of any signal is enclosed in brackets (e.g., $x[k]=$ $x(k T)$ ), and where the equations relating the continuoustime system (1) to the discrete-time equivalent model (2) depend on the sampling period and holding process. Assume now that a simple $\mathrm{ZOH}$ is applied to all control agents. The discrete-time equivalent model (2), in this case, assuming $A$ is invertible, is described by the following matrices:

$$
A_{d}=e^{A T}, b_{d_{j}}=A^{-1}\left(A_{d}-I\right) b_{j}, c_{d_{j}}=c_{j},
$$

for any $j \in \overline{\mathrm{m}}$. A controller with output $u[k]$ can now be constructed from $y[i], i \leq k$.

Consider now applying a more general form of hold function to the system (1), instead of a simple ZOH. Generalized sampled-data hold functions (GSHF) can be formulated as follows:

$$
\begin{aligned}
& u_{j}(t)=f_{j}(t) \tilde{u}_{j}[k], \quad t \in[k T,(k+1) T), \quad k=0,1,2, \ldots \\
& f_{j}(t+T)=f_{j}(t),
\end{aligned}
$$

for any $j \in \overline{\mathrm{m}}$. The discrete-time equivalent model (2), in this case, is described by the following matrices:

$$
\begin{aligned}
A_{d} & =e^{A T}, \\
b_{d_{j}} & =\int_{0}^{T} e^{A(T-\tau)} b_{j} f_{j}(\tau) d \tau, \\
c_{d_{j}} & =c_{j},
\end{aligned}
$$

for any $j \in \overline{\mathrm{m}}$. It was shown in (Willems 1988) that if $\lambda_{1}$ is a nonzero unstructured DFM of the original continuous-time system and a cyclic eigenvalue of the system matrix $A$ (i.e., the Jordan block corresponding to $\lambda_{1}$ has maximal dimension), it will no longer be a DFM of the sampled system for almost all sampling periods. A condition will now be given, under which a continuoustime feedback followed by sampling can eliminate any nonzero unstructured DFMs which are repeated noncyclic eigenvalues of the system matrix $A$.

Theorem 1 Consider the $n^{\text {th }}$ order continuous-time decentralized system (1). For simplicity and without loss of generality, assume that each control agent is singleinput single-output (SISO), i.e., $u_{i}(t), y_{i}(t) \in \mathbb{R}, i \in \overline{\mathrm{m}}$. Assume also that $\lambda_{1} \neq 0$ is an unstructured DFM of the system and a repeated non-cyclic eigenvalue of the 
system matrix $A$ (which implies that $\operatorname{rank}\left(A-\lambda_{1} I\right)<$ $n-1)$. Then the fixed mode $\lambda_{1}$ can be eliminated by applying a continuous-time decentralized LTI output feedback $u_{i}(t)=K_{i} y_{i}(t), i \in \overline{\mathrm{m}}$ followed by ZOH sampling, for almost all sampling periods $T$ and almost all feedback gains $K_{1}, \ldots, K_{m}$, if for any partition $I_{1}=\left\{i_{1}, \ldots, i_{k}\right\}$ and $I_{2}=\left\{i_{k+1}, \ldots, i_{m}\right\}$ of the set $\overline{\mathrm{m}}=\{1, \ldots, m\}$, so that $I_{1} \cup I_{2}=\overline{\mathrm{m}}$ and $I_{1} \cap I_{2}=\emptyset$, the following condition holds:

$$
\operatorname{rank}\left(\left[\begin{array}{cccc}
A-\lambda_{1} I & b_{i_{1}} & \ldots & b_{i_{k}} \\
c_{i_{k+1}} & 0 & \ldots & 0 \\
\vdots & \vdots & & \vdots \\
c_{i_{m}} & 0 & \ldots & 0
\end{array}\right]\right) \geq n-1
$$

Proof of Theorem 1 From (Anderson and Clements 1981), condition (6) is equivalent to the condition that there exist $K_{1}, \ldots, K_{m}$ so that:

$$
\operatorname{rank}\left(A+b_{1} K_{1} c_{1}+\ldots+b_{m} K_{m} c_{m}-\lambda_{1} I\right) \geq n-1
$$

and if condition (7) holds, this implies it must hold for almost all $K_{1}, \ldots, K_{m}$. It follows immediately from Proposition 2 in (Willems 1988) that the discrete-time system obtained by sampling the resultant continuous-time closed-loop system does not have a DFM for almost all sampling periods $T$.

Definition 3 Consider the system (1). A partition of the system consisting of l subsystems $\left(\bar{c}_{1}, A, \bar{b}_{1}\right),\left(\bar{c}_{2}, A, \bar{b}_{2}\right)$, $\ldots,\left(\bar{c}_{l}, A, \bar{b}_{l}\right),(1 \leq l \leq m)$, where:

$$
\begin{aligned}
& \bar{c}_{1}=\left[\begin{array}{c}
c_{i_{1}} \\
c_{i_{2}} \\
\vdots \\
c_{i_{k_{1}}}
\end{array}\right], \bar{c}_{2}=\left[\begin{array}{c}
c_{i_{k_{1}+1}} \\
c_{i_{k_{1}+2}} \\
\vdots \\
c_{i_{k_{2}}}
\end{array}\right], \ldots, \bar{c}_{l}=\left[\begin{array}{c}
c_{i_{k_{l-1}+1}} \\
c_{i_{k_{l-1}+2}} \\
\vdots \\
c_{i_{k_{l}}}
\end{array}\right] \\
& \bar{b}_{1}=\left[\begin{array}{llll}
b_{i_{1}} & b_{i_{2}} & \ldots & b_{i_{k_{1}}}
\end{array}\right], \bar{b}_{2}=\left[\begin{array}{llll}
b_{i_{k_{1}+1}} & b_{i_{k_{1}+2}} & \ldots & b_{i_{k_{2}}}
\end{array}\right], \ldots, \\
& \bar{b}_{l}=\left[\begin{array}{llll}
b_{i_{k_{l-1}+1}} & b_{i_{k_{l-1}+2}} & \ldots & b_{i_{k_{l}}}
\end{array}\right] \text {, } \\
& I_{1}=\left\{i_{1}, \ldots, i_{k_{1}}\right\}, I_{2}=\left\{i_{k_{1}+1}, \ldots, i_{k_{2}}\right\}, \ldots \\
& I_{l}=\left\{i_{k_{l-1}+1}, \ldots, i_{k_{l}}\right\},(8 \mathrm{c}) \\
& I_{1} \cup I_{2} \cup \ldots \cup I_{l}=\{1,2, \ldots, m\}, I_{1} \cap I_{2} \cap \ldots \cap I_{l}=\emptyset \text {, }
\end{aligned}
$$

is called a partition of size l for the system (1).

It can be concluded from the definition of a strongly connected system that a partition of size $l$ given by (8) for the system (1) represents a quotient system if and only if there exist no subsets of $I_{j}$ denoted by $I_{j}^{1}=\left\{p_{j}^{1}, \ldots, p_{j}^{r_{1}}\right\}$,
$I_{j}^{2}=\left\{p_{j}^{r_{1}+1}, \ldots, p_{j}^{r_{2}}\right\}, j=1,2, \ldots, l$, where $I_{j}^{1} \cup I_{j}^{2}=I_{j}$ and $I_{j}^{1} \cap I_{j}^{2}=\emptyset$, such that:

$$
\left[\begin{array}{c}
c_{p_{j}^{r_{1}+1}} \\
\vdots \\
c_{p_{j}^{r_{2}}}
\end{array}\right](s I-A)^{-1}\left[b_{p_{j}^{1}} \ldots b_{p_{j}^{r_{1}}}\right]=0, \quad \forall s \in \mathbb{C}
$$

The following initial result is obtained.

Theorem 2 Consider the system (1); then $\lambda \in \operatorname{sp}(A)$ is a QFM of the system if and only if there exists an integer $l$ such that $\lambda$ is a transmission zero of all of the following subsystems:

$$
\left(\left[\begin{array}{llll}
\hat{c}_{1}^{\prime} & \hat{c}_{2}^{\prime} & \ldots & \hat{c}_{l}^{\prime}
\end{array}\right]^{\prime}, A,\left[\begin{array}{llll}
\hat{b}_{1} & \hat{b}_{2} & \ldots & \hat{b}_{l}
\end{array}\right]\right),
$$

where the rows of $\hat{c}_{i}$ and the columns of $\hat{b}_{i}$ consist of any subset of the rows of $\bar{c}_{i}$ and columns of $\bar{b}_{i}$ given by (8a), (8b) (including empty sets) respectively $(i=1,2, \ldots, l)$ such that the number of rows of $\hat{c}_{i}$ is equal to the number of columns of $\hat{b}_{i}, i=1,2, \ldots, l$.

Proof of Theorem 2 The quotient system corresponding to the system (1) has the general structure of Figure 3. In this case, the bidirectional arcs represent two separate edges in two opposite directions between the corresponding nodes, $C_{i}, i=1,2, \ldots, l$ represent the control agents corresponding to the strongly connected subsystems and $i_{k_{1}}, \ldots, i_{k_{l}}$ are introduced in (8). The edges with no arrays in this figure represent unidirectional arcs. It is to be noted that unidirectional edges can also exist between the nodes of the disjoint strongly connected subsystems, as long as they do not create a loop between the corresponding nodes. Here each controller $C_{i}$ has a centralized struc-

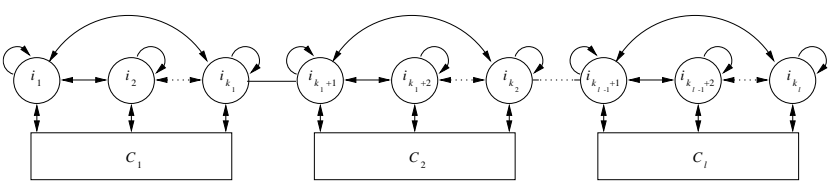

Fig. 3. The general form of the quotient system for system (1).

ture. This implies that there can exist feedback from each node of the subsystem (which represents the inputs and outputs of the respective agent) to any other node of the same subsystem via the controller $C_{i}$. Hence, $\lambda \in \operatorname{sp}(A)$ is a QFM of the system (1) if and only if it is a DFM of the system with respect to the block diagonal matrix:

$$
K=\operatorname{diag}\left(K_{1}, K_{2}, \ldots, K_{l-1}, K_{l}\right)
$$

where $K_{i}(i=1,2, \ldots, l)$ is a $\left(k_{i}-k_{i-1}\right) \times\left(k_{i}-k_{i-1}\right)$ gain matrix $\left(k_{0} \triangleq 0\right)$. It can be concluded from the structure of 
the quotient system, that $\lambda$ is a QFM of the system (1) if and only if it is a DFM of the following system (Davison and Chang 1990):

$$
\begin{aligned}
& \dot{x}=A x+B u, \\
& y=C x,
\end{aligned}
$$

with the $B$ and $C$ matrices given below:

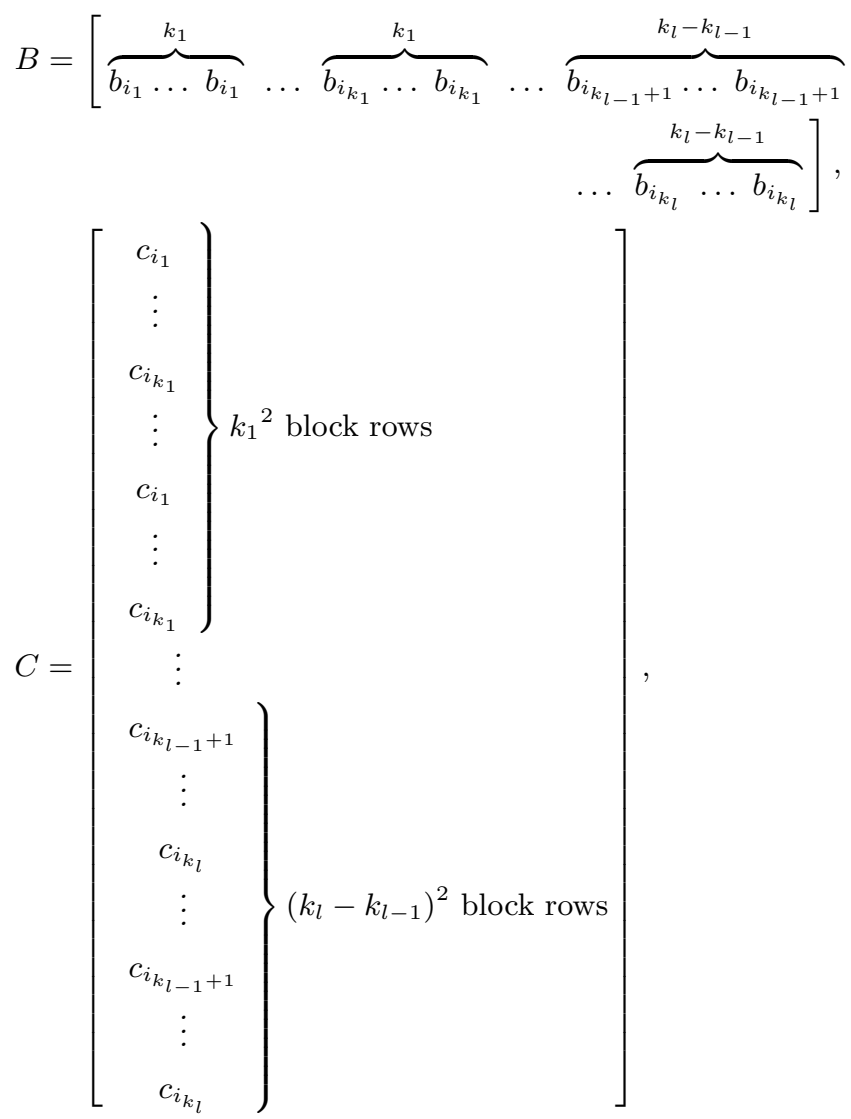

and so, the application of Theorem 2 of (Davison and Chang 1990) immediately leads to the proof of Theorem 2.

Proposition 1 Consider the system (1) consisting of $l$ strongly connected subsystems; then $\lambda \in \operatorname{sp}(A)$ is a $Q F M$ of the system (1) if and only if for the partition:

$$
\left(\left[\begin{array}{llll}
\bar{c}_{1}^{\prime} & \bar{c}_{2}^{\prime} & \ldots & \bar{c}_{l}^{\prime}
\end{array}\right]^{\prime}, A,\left[\begin{array}{llll}
\bar{b}_{1} & \bar{b}_{2} & \ldots & \bar{b}_{l}
\end{array}\right]\right)
$$

representing a quotient system for (1), the following con- dition holds:

$$
\operatorname{rank}\left(\left[\begin{array}{cccc}
A-\lambda I & \bar{b}_{q_{1}} \ldots & \bar{b}_{q_{k}} \\
\bar{c}_{q_{k+1}} & 0 & \ldots & 0 \\
\vdots & \vdots & & \vdots \\
\bar{c}_{q_{l}} & 0 & \ldots & 0
\end{array}\right]\right)<n
$$

for some subsets $\left\{q_{1}, \ldots, q_{k}\right\}$ and $\left\{q_{k+1}, \ldots, q_{l}\right\}$ of $\{1,2, \ldots, l\}$, such that $\left\{q_{1}, \ldots, q_{k}\right\} \cup\left\{q_{k+1}, \ldots, q_{l}\right\}=$ $\{1,2, \ldots, l\}$ and $\left\{q_{1}, \ldots, q_{k}\right\} \cap\left\{q_{k+1}, \ldots, q_{l}\right\}=\emptyset$.

Proof of Proposition 1 The proof follows immediately on applying the criterion of (Anderson and Clements 1981) to the decentralized structure of the corresponding quotient system.

Theorem 3 Consider the system (1), and suppose that the system has distinct and nonzero modes. Let the corresponding decoupled model be given by:

$$
\begin{aligned}
\dot{\check{x}}(t) & =\Lambda \check{x}(t)+\sum_{j=1}^{m} \check{b}_{j} u_{j}(t), \\
y_{j}(t) & =\check{c}_{j} \check{x}(t),
\end{aligned}
$$

where $j \in \overline{\mathrm{m}}$, and $\Lambda$ is a diagonal matrix consisting of the eigenvalues of the system. For simplicity and without loss of generality assume that $m=2$, and that the system has two strongly connected subsystems $\left(\bar{c}_{1}, \Lambda, \bar{b}_{1}\right),\left(\bar{c}_{2}, \Lambda, \bar{b}_{2}\right)$, where $\bar{b}_{1}, \bar{b}_{2}, \bar{c}_{1}, \bar{c}_{2}$ are defined in (8). Moreover, suppose that $\lambda_{1}$ is an observable and controllable DFM of the system. Then $\lambda_{1}$ is a structured DFM if and only if it is a QFM of this system.

Proof of Theorem $3 \lambda_{1}$ is a QFM of (1) if and only if the following conditions both hold:

i) $\lambda_{1}$ is a DFM of the system consisting of the two subsystems 1 and 2;

ii) the two subsystems themselves are not strongly connected.

It can be concluded from (Özgüner and Davison 1985) (generalization of Lemma 3) that condition (i) holds if and only if either conditions (a) and (b) or conditions (c) and (d) given below hold:

a) $\bar{b}_{1}^{1}=0$ and $\bar{c}_{2}^{1}=0$

b) $\left[\begin{array}{llll}\bar{c}_{2}^{2} & \bar{c}_{2}^{3} & \ldots & \bar{c}_{2}^{n}\end{array}\right]\left[\begin{array}{cccc}\lambda_{2}-\lambda_{1} & 0 & \ldots & 0 \\ 0 & \lambda_{3}-\lambda_{1} & \ldots & 0 \\ \vdots & \vdots & & \vdots \\ 0 & 0 & \ldots & \lambda_{n}-\lambda_{1}\end{array}\right]^{-1}\left[\begin{array}{c}\bar{b}_{1}^{2} \\ \bar{b}_{1}^{3} \\ \vdots \\ \bar{b}_{1}^{n} \\ =0\end{array}\right]$ 
c) $\bar{b}_{2}^{1}=0$ and $\bar{c}_{1}^{1}=0$

d) $\left[\begin{array}{llll}\bar{c}_{1}^{2} & \bar{c}_{1}^{3} & \ldots & \bar{c}_{1}^{n}\end{array}\right]\left[\begin{array}{cccc}\lambda_{2}-\lambda_{1} & 0 & \ldots & 0 \\ 0 & \lambda_{3}-\lambda_{1} & \ldots & 0 \\ \vdots & \vdots & & \vdots \\ 0 & 0 & \ldots & \lambda_{n}-\lambda_{1}\end{array}\right]^{-1}\left[\begin{array}{c}\bar{b}_{2}^{2} \\ \bar{b}_{2}^{3} \\ \vdots \\ \bar{b}_{2}^{n} \\ =0\end{array}\right]$

where $\bar{b}_{j}^{i}$ and $\bar{c}_{j}^{i}, j=1,2, i=1, \ldots, n$, represent row $i$ of $\bar{b}_{j}$ and column $i$ of $\bar{c}_{j}$, respectively.

Condition (ii) holds if and only if either

$\left[\begin{array}{llll}\bar{c}_{2}^{1} & \bar{c}_{2}^{2} & \ldots & \bar{c}_{2}^{n}\end{array}\right]\left[\begin{array}{cccc}s-\lambda_{1} & 0 & \ldots & 0 \\ 0 & s-\lambda_{2} & \ldots & 0 \\ \vdots & \vdots & & \vdots \\ 0 & 0 & \ldots & s-\lambda_{n}\end{array}\right]^{-1}\left[\begin{array}{c}\bar{b}_{1}^{1} \\ \bar{b}_{1}^{2} \\ \vdots \\ \bar{b}_{1}^{n}\end{array}\right]=0$

or:

$\left[\begin{array}{llll}\bar{c}_{1}^{1} & \bar{c}_{1}^{2} & \ldots & \bar{c}_{1}^{n}\end{array}\right]\left[\begin{array}{cccc}s-\lambda_{1} & 0 & \ldots & 0 \\ 0 & s-\lambda_{2} & \ldots & 0 \\ \vdots & \vdots & & \vdots \\ 0 & 0 & \ldots & s-\lambda_{n}\end{array}\right]^{-1}\left[\begin{array}{c}\bar{b}_{2}^{1} \\ \bar{b}_{2}^{2} \\ \vdots \\ \bar{b}_{2}^{n}\end{array}\right]=0$

for all $s \in \mathbb{C}$. This implies that condition (ii) holds if and only if either condition (e) or condition ( $f$ ) given below holds:

e) $\bar{c}_{2}^{i} \bar{b}_{1}^{i}=0, i=1, \ldots, n$

f) $\bar{c}_{1}^{i} \bar{b}_{2}^{i}=0, i=1, \ldots, n$

It can be easily verified that condition (b) follows from condition (e) and condition (d) follows from condition (f). On the other hand, because of the controllability assumption $b_{1}^{1}$ and $b_{2}^{1}$ both cannot be zero and because of observability assumption $c_{1}^{1}$ and $c_{2}^{1}$ both cannot be zero (note that a column vector times a row vector is equal to a zero matrix if and only if at least one of the vectors is zero). This implies that:

- conditions (a) and (f) cannot both hold,

- conditions (c) and (e) cannot both hold.

Thus, it can be concluded that $\lambda_{1}$ is a QFM of (1) if and only if either condition (iii) or condition (iv) given below holds:

iii) $\bar{b}_{1}^{1}=0$ and $\bar{c}_{2}^{1}=0$ and $\bar{c}_{2}^{i} \bar{b}_{1}^{i}=0, i=1, \ldots, n$.

iv) $\bar{b}_{2}^{1}=0$ and $\bar{c}_{1}^{1}=0$ and $\bar{c}_{1}^{i} b_{2}^{i}=0, i=1, \ldots, n$.
It follows immediately from (Özgüner and Davison 1985) (generalization of Lemma 3) that $\lambda_{1}$ is a QFM if and only if it is a structured DFM of the system.

Remark 1 The results of Theorem 3 can be directly extended to systems with more than two interconnected subsystems. This implies that a DFM which is nonzero and distinct is a structured DFM if and only if it is a QFM.

\subsection{Effect of Sampling on ADFMs}

A brief description of approximate decentralized fixed modes (ADFM) as proposed in (Vaz and Davison 1989) will now be given. Consider the system (1), and for simplicity assume that the system consists of two SISO subsystems. In this case, $\lambda \in \operatorname{sp}(A)$ is a DFM if and only if it is a transmission zero of all of the following three systems (Davison and Chang 1990):

$$
\left(c_{1}, A, b_{1}\right),\left(c_{2}, A, b_{2}\right),\left(\left[\begin{array}{l}
c_{1} \\
c_{2}
\end{array}\right], A,\left[\begin{array}{ll}
b_{1} & b_{2}
\end{array}\right]\right)
$$

Now, let the condition measure of a matrix $M$ which gives a measure of how "close" it is to being singular, be defined as $1 / \rho(M)$, where $\rho(M)$ denotes the smallest singular value of $M$; it is to be noted that other measures could also be chosen, but for simplicity the above will be used. Now, let the condition measures of the following three matrices:

$$
\left[\begin{array}{rr}
A-\lambda I & b_{1} \\
c_{1} & 0
\end{array}\right],\left[\begin{array}{lr}
A-\lambda I & b_{2} \\
c_{2} & 0
\end{array}\right],\left[\begin{array}{lrr}
A-\lambda I & b_{1} & b_{2} \\
c_{1} & 0 & 0 \\
c_{2} & 0 & 0
\end{array}\right],
$$

be denoted by $\operatorname{cond}_{1}(\lambda), \operatorname{cond}_{2}(\lambda)$ and $\operatorname{cond}_{1,2}(\lambda)$, respectively, where $\lambda \in \operatorname{sp}(A)$, and define:

$$
\operatorname{cond}(\lambda)=\min \left\{\operatorname{cond}_{1}(\lambda), \operatorname{cond}_{2}(\lambda), \operatorname{cond}_{1,2}(\lambda)\right\} .
$$

Definition $4 \lambda$ is said to be a mode of magnitude $\operatorname{cond}(\lambda)$ given above, and in this case, the larger cond $(\lambda)$ is, the closer $\lambda$ is to being a DFM (i.e., $\lambda$ is a DFM if and only if $\operatorname{cond}(\lambda)=\infty)$. If $\operatorname{cond}(\lambda)$ is "large", then the system is said to have an approximate DFM (ADFM) of magnitude cond $(\lambda)$ at $\lambda$. A similar definition of $A D F M$ can be made when the number of control agents is greater than 2, and also when the subsystems are multi-input multi-output (MIMO), using the results of (Davison and Chang 1990) and (Vaz and Davison 1989).

As discussed earlier, if the continuous-time system (1) has distinct and nonzero unstructured DFMs, then for generic sampling times $T>0$ there exist no unstructured DFMs (Özgüner and Davison 1985) in the 
discrete-time equivalent model (2) obtained by using any nonzero hold function (ZOH or GSHF). There may, however, exist ADFMs for these respective modes, and since as the sampling time approaches zero, the behavior of the discrete-time system converges to the continuous-time system, this implies that for sufficiently small sampling periods, the discrete-time system will have ADFMs which tend to infinity as $T \rightarrow 0$.

Remark 2 It can be concluded from the above discussion that to avoid large ADFMs, one should choose sufficiently large sampling periods. On the other hand, a large sampling period makes the control signal sluggish. More precisely, it is often desired to choose a sampling frequency twice as large as the bandwidth of the sampled signal in order to avoid excessive aliasing in the control process. This introduces a trade-off in the size of the sampling interval, and thus the choice of an "optimal" sampling period involves an optimization problem.

A method will now be given to measure the magnitude of an ADFM of a discrete-time systems using an approach similar to the continuous-time case. Consider the discrete-time model (2) and for simplicity, without loss of generality, assume that each control agent has a scalar input and a scalar output, i.e., $u_{i}(t), y_{i}(t) \in \mathbb{R}, i \in \overline{\mathrm{m}}$. Subtracting $x[k]$ from both sides of (2a) and dividing the resultant equation by $T$, the following equation will be obtained:

$$
\frac{x[k+1]-x[k]}{T}=\left(\frac{A_{d}-I}{T}\right) x[k]+\left[\frac{b_{d_{1}}}{T} \ldots \frac{b_{d_{m}}}{T}\right] u[k] .
$$

Equation (12) is an approximation to the original continuous-time equation (1a). It can be verified that as $T \rightarrow 0$, the solution of equation (12) approaches the solution of (1a). In this case, if $\lambda$ is contained in $\operatorname{sp}(A)$, then $\frac{e^{\lambda T}-1}{T}$ is an eigenvalue of $\frac{A_{d}-I}{T}$.

Definition 5 Given $\lambda$ contained in $\operatorname{sp}(A)$, let $\operatorname{cond}_{d}\left(e^{\lambda T}\right)$ denote the minimum condition measure of the following matrices:

$$
\left[\begin{array}{cccc}
\frac{e^{A T}-e^{\lambda T} I}{T} & \frac{b_{d_{i_{1}}}}{T} & \ldots & \frac{b_{d_{i_{k}}}}{T} \\
c_{d_{i_{1}}} & 0 & \ldots & 0 \\
\vdots & \vdots & & \vdots \\
c_{d_{i_{k}}} & 0 & \ldots & 0
\end{array}\right]
$$

where $i_{1}, \ldots, i_{k}$ denote any distinct integers so that $\left\{i_{1}, \ldots, i_{k}\right\} \subset \overline{\mathrm{m}}$. In this case, $\operatorname{cond}_{d}\left(e^{\lambda T}\right)$ gives a measure of the magnitude of an ADFM for the discrete-time equivalent model. Note that for a given matrix $A$ with $\lambda \in \operatorname{sp}(A)$, cond $_{d}\left(e^{\lambda T}\right)$ converges to $\operatorname{cond}(\lambda)$ as the sampling interval $T$ approaches zero.
Consider now the continuous-time decentralized system (1). Assume that $\lambda \in \operatorname{sp}(A)$ is an ADFM of magnitude $\operatorname{cond}(\lambda)=M$. Let the discrete-time equivalent representation of (1) for a sampling period $T$ and a given hold function be represented by (2) and (3); then if $\operatorname{cond}\left(e^{\lambda T}\right) \ll M$, this implies that $\lambda$ is closer to being a DFM for the original continuous-time system (1) as compared to $e^{\lambda T}$ for the discrete-time equivalent model (3). In other words, a digital controller with a simple $\mathrm{ZOH}$, can potentially improve the performance of a continuous-time closed-loop system, when there exists an unstructured ADFM in the continuous-time system.

Remark 3 On applying the approach given in Definition 4 to the quotient system, one can define an approximate quotient fixed mode (AQFM). It can be observed that a structured ADFM is, in fact, an AQFM (the opposite, however, is not necessarily true). Note that if $\lambda \in \operatorname{sp}(A)$ is an ADFM of "large" magnitude and an AQFM of "small" magnitude, this implies that the corresponding mode is close to being a DFM but not close to being a QFM. In other words, it is easier to shift this mode by applying a proper time-varying control law rather than a conventional LTI controller.

It is desired now to show how GSHFs can improve the performance of the closed-loop system.

Lemma 1 Consider the system (1) with sampleddata hold functions (4). For any sampling period $T$, and any arbitrary $n \times s_{j}$ nonzero matrix $b_{j}^{*}, j \in \overline{\mathrm{m}}$, whose columns belong to the controllable subspace of $\left(A, b_{j}\right)$, there exists a $s_{j} \times s_{j} G S H F f_{j}$ so that with $u_{j}(t)=f_{j}(t) \tilde{u}_{j}[k], t \in[k T,(k+1) T)$, the corresponding discrete-time system (2), has the property that $b_{d_{j}}=b_{j}^{*}$.

Proof of Lemma 1 The proof follows by using an argument similar to that presented in (Kabamba 1987). Define:

$\tilde{b}_{d_{j}}(t):=\int_{t_{k}}^{t} e^{A(t-\tau)} b_{j} f_{j}(\tau) d \tau$.

Taking the derivative of the above equation results in

$$
\begin{aligned}
\frac{d}{d t} \tilde{b}_{d_{j}}(t) & =e^{A(t-t)} b_{j} f_{j}(t)+\int_{t_{k}}^{t} A e^{A(t-\tau)} b_{j} f_{j}(\tau) d \tau \\
& =A \tilde{b}_{d_{j}}(t)+b_{j} f_{j}(t)
\end{aligned}
$$

The above set of differential equations can be written for any column of $\tilde{b}_{d_{j}}(t)$ and $f_{j}(t), j \in \overline{\mathrm{m}}$ separately. Each of these differential equations can then be considered as a state-space model, whose input is an appropriate column of $f_{j}(t)$. Therefore, if $b^{* i}{ }_{j}\left(i \in\left\{1,2, \ldots, s_{j}\right\}, j \in \overline{\mathrm{m}}\right)$ is in the controllable subspace of $\left(A, b_{j}\right)$, for any $T>0$, there exists a $s_{j} \times 1$ function $f_{j}^{i}(t)$ that can transform $\tilde{b}_{d_{j}}^{i}\left(t_{k}\right)=$ 
0 to $\tilde{b}_{d_{j}}^{i}\left(t_{k+1}\right)=b_{j}^{* i}$, where $f_{j}^{i}(t), \tilde{b}_{d_{j}}^{i}\left(t_{k}\right)$ and $b^{* i}{ }_{j}$ denote the ith column of $f_{j}(t), \tilde{b}_{d_{j}}\left(t_{k}\right)$ and $b^{*}{ }_{j}$ respectively, and $t_{k+1}-t_{k}=T$. In other words, one can find a $s_{j} \times s_{j}$ $G S H F$, so that the matrix $b_{d_{j}}$ in the resulting discretetime system has the desired form.

Lemma 1 states that one can always find a solution to the following problem:

Problem 1: Find a $s_{j} \times s_{j}$ GSHF $f_{j}$ (non-unique), so that the matrix $b_{d_{j}}$ in the resultant discrete-time system is equal to any specified $n \times s_{j}$ matrix whose columns belong to the controllable subspace of $\left(A, b_{j}\right), j \in \overline{\mathrm{m}}$.

In fact, one can use the following steps to find a possible solution to the above problem:

i) Assume that the rank of the controllable subspace of $\left(A, b_{j}\right)$ is equal to $n_{1}$, for some $n_{1} \leq n$. Find a transformation $V$ to partition the system as:

$$
\begin{aligned}
& \bar{A}:=V^{-1} A V=\left[\begin{array}{ll}
\bar{A}_{11_{\left(n_{1} \times n_{1}\right)}} & \bar{A}_{12_{\left(n_{1} \times\left(n-n_{1}\right)\right)}} \\
0_{\left(\left(n-n_{1}\right) \times n_{1}\right)} & \bar{A}_{22_{\left(\left(n-n_{1}\right) \times\left(n-n_{1}\right)\right)}}
\end{array}\right] \\
& \bar{b}_{j}:=V^{-1} b_{j}=\left[\begin{array}{l}
\bar{b}_{j 1_{\left(n_{1} \times s_{j}\right)}} \\
0_{\left(\left(n-n_{1}\right) \times s_{j}\right)}
\end{array}\right],
\end{aligned}
$$

where the controllable pair $\left(\bar{A}_{11}, \bar{b}_{j 1}\right)$ represents the controllable subspace of $\left(A, b_{j}\right)$. The first $n_{1}$ columns of $V$ can be any set of vectors that span the column space of the controllability matrix $\left[b_{j} A b_{j} \ldots A^{n-1} b_{j}\right]$, and the last $n-n_{1}$ columns can be any set of vectors that results in a nonsingular matrix $V$.

ii) Find the controllability Gramian corresponding to $\left(\bar{A}_{11}, \bar{b}_{j 1}\right)$ on $[0, T]$ as follows:

$$
\bar{W}_{T, j}=\int_{0}^{T} e^{t \bar{A}_{11}} \bar{b}_{j 1} \bar{b}_{j 1}^{\prime} e^{t \bar{A}_{11}^{\prime}} d t .
$$

iii) Then, the following GSHF is a solution (non-unique) to Problem 1:

$$
f_{j}(t)=\bar{b}_{j 1}^{\prime} e^{(T-t) \bar{A}_{11}^{\prime}} \bar{W}_{T, j}^{-1} \bar{b}_{j 1}^{*},
$$

where $\bar{b}_{j 1}^{*}$ is obtained from the following equation:

$$
\bar{b}_{j}^{*}:=V^{-1} b_{j}^{*}=\left[\begin{array}{l}
\bar{b}_{j 1_{\left(n_{1} \times s_{j}\right)}^{*}} \\
0_{\left(\left(n-n_{1}\right) \times s_{j}\right)}
\end{array}\right] .
$$

Consider now the system (1) and assume that $\lambda \in \operatorname{sp}(A)$ is an unstructured ADFM of large magnitude. It is desired to find the matrices $b_{j}^{*}$ in the controllable subspace of $\left(A, b_{j}\right), j \in \overline{\mathrm{m}}$, such that the magnitude of the $\mathrm{ADFM} e^{\lambda T}$ in the discrete-time system $\left(\left[\begin{array}{ccc}c_{1}^{\prime} & \ldots & c_{m}^{\prime}\end{array}\right]^{\prime}\right.$, $\left.e^{A T},\left[b_{1}^{*} \ldots b_{m}^{*}\right]\right)$ is minimized. One can use the result of Lemma 1 and the procedure given above to find a GSHF which leads to the matrices $b_{j}^{*}, j \in \overline{\mathrm{m}}$ in the discrete-time equivalent model, and hence minimizes the magnitude of the corresponding ADFM.

Given a continuous-time system which has a large ADFM and a small AQFM, it is to be noted that if the discrete-time equivalent model obtained by a $\mathrm{ZOH}$ has an ADFM of large magnitude, then a dynamic discrete-time controller of any order is not necessarily most effective in improving the overall performance, compared to a GSHF controller. In other words, introducing more degrees of freedom by deploying a higher order dynamic controller with a simple $\mathrm{ZOH}$ may not be as effective as introducing more degrees of freedom to the GSHF controller, as far as the performance of the overall decentralized control system is concerned.

Remark 4 One can apply simple functions, e.g., the class of polynomials of specific degree, instead of the function defined in equation (13), in order to minimize the condition measure of an ADFM. For example, by using the class of second-order polynomial functions, one can minimize the condition measure over $3 \sum_{j=1}^{m} s_{j}^{2}$ parameters (note that each second-order function includes three coefficients). Alternately, one could also apply piecewise constant functions, instead of polynomial functions, to create a GSHF. The optimal choice of the GSHF (i.e., the choice of the proper order and coefficients in a polynomial GSHF for a given system) is an open question for research, and can be tackled by using the "finite set of basis functions" approach proposed in (Lavaei and Aghdam 200\%) and (Lavaei and Aghdam 200\%).

Remark 5 It is to be noted that in practice all DFMs are structured and there is no unstructured DFM, since unstructured DFMs result from an exact matching of plant parameters, which is very unlikely to happen in physical applications (this point will be further clarified in Example 1). However, in practice, a close matching of parameters of a system may cause ADFMs, for which the proposed discrete-time decentralized controller can outperform the continuous-time counterpart.

The results obtained for DFMs, QFMs, ADFMs and AQFMs of (1) can be summarized as follows. Using a sampled-data controller:

- One can eliminate a nonzero non-repeated unstructured DFM and eliminate a nonzero non-repeated ADFM which is not an AQFM (Remark 3 in present paper); (Özgüner and Davison 1985); (Willems 1988).

- One cannot eliminate a structured DFM and cannot eliminate an ADFM which is an AQFM (Remark 3 in present paper); (Özgüner and Davison 1985). 
- A mode which is an unstructured DFM of the system and a repeated non-cyclic eigenvalue of the $A$-matrix in the state-space representation can be eliminated by applying a continuous-time decentralized LTI output feedback followed by sampling, provided the system matrices satisfy a mild condition (Theorem 1 in present paper).

- A zero DFM cannot be eliminated (Willems 1988).

In the next section, it will be shown how the control analysis introduced so far can be efficiently used in the design of GSHFs, in order to improve the overall performance of the system, in the presence of an unstable unstructured ADFM.

\section{Numerical Example}

Example 1 Consider a continuous-time controllable, observable, non-minimum phase, unstable system with the following state-space matrices:

$$
\begin{aligned}
& A=\left[\begin{array}{ccc}
-1 & 0 & -3 \\
0 & 0.1 & 0 \\
0 & 0 & -3
\end{array}\right], b_{1}=\left[\begin{array}{l}
1 \\
0 \\
1
\end{array}\right], b_{2}=\left[\begin{array}{l}
0 \\
1 \\
1
\end{array}\right], \\
& c_{1}=\left[\begin{array}{lll}
0 & 1 & 0
\end{array}\right], c_{2}=\left[\begin{array}{lll}
-1.1 & 0.005 & 0.1
\end{array}\right] .
\end{aligned}
$$

This is the example given in (Aghdam and Davison 1999). The eigenvalues of this system are $\operatorname{sp}(A)=$ $\{-1,0.1,-3\}$. Applying the minimum condition measure criterion of Section 2.3 to this system results in:

$\operatorname{cond}(-1)=1.762, \operatorname{cond}(0.1)=400.0, \operatorname{cond}(-3)=1.186$

and so, $\lambda=0.1$ can be considered as being a large $A D F M$ for the system. However, this mode is not a large AQFM as can be verified by applying Definition 4 to the corresponding quotient system. To illustrate this, let the second entry of $c_{2}$ (i.e. 0.005) be replaced by a zero, to obtain the perturbed matrix $\tilde{c}_{2}=\left[\begin{array}{lll}-1.1 & 0 & 0.1\end{array}\right]$. From Theorems 2 and 3, one can conclude that $\lambda=0.1$ is a DFM of the resultant system, but not a QFM. More precisely, the following matrix:

$$
\left[\begin{array}{lr}
A-0.1 I & b_{1} \\
\tilde{c}_{2} & 0
\end{array}\right]=\left[\begin{array}{cccc}
-1.1 & 0 & -3 & 1 \\
0 & 0 & 0 & 0 \\
0 & 0 & -3.1 & 1 \\
-1.1 & 0 & 0.1 & 0
\end{array}\right]
$$

has rank equal to 2 due to the perfect matching of the nonzero parameters, which confirms that this mode is an unstructured DFM. Thus, any perturbation in the parameters of the system will eliminate this fixed mode and if the perturbation is sufficiently small, the mode will be an unstructured $A D F M$. This indeed confirms the finding that $\lambda=0.1$ is an unstructured ADFM of the system (14) with a large magnitude of 400.0. Note that this is an unstable mode and so to stabilize the system, the decentralized continuous-time LTI controller has to "shift" it to the left half plane, which implies that it will require a large gain. On applying the minimum condition measure criterion for ADFM in the discrete-time equivalent model obtained by using a simple $\mathrm{ZOH}$, the results shown in Figure 4 are obtained. These results give the minimum condition measure for each eigenvalue of the discrete-time equivalent model as a function of the sampling period. It can be seen from these results, that for a wide range of sampling intervals, the discrete-time equivalent model has no ADFM of large magnitude. For instance, with a sampling interval of $T=2.9 \mathrm{sec}$, the condition measure of the ADFM $e^{0.1 T}$ for the discrete-time equivalent model is 151.8 which is approximately 3 times smaller than cond $(0.1)=400.0$ for the continuous-time system. As a rule of thumb, if the minimum condition measures associated with the unstable modes in the continuous-time system are significantly greater than their discrete-time counterparts, a suitable digital controller can potentially be more effective than a LTI one.
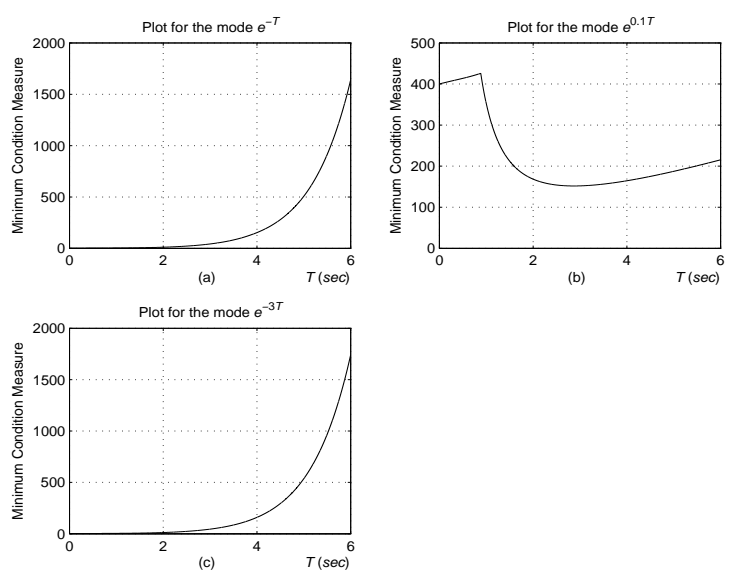

Fig. 4. Minimum condition measures of Example 1 versus sampling period for each eigenvalue of the discrete-time equivalent model. (a) $e^{-T}$, (b) $e^{0.1 T}$, (c) $e^{-3 T}$.

It is desired now to find a controller to stabilize the unstable system (14), and the following performance index is considered as a measure of the system performance using either continuous-time or discrete-time static decentralized controllers:

$$
J=\mathcal{E}\left\{\int_{0}^{\infty}\left(y^{\prime} y+u^{\prime} u\right) d t\right\}
$$

where $\mathcal{E}$ denotes the expectation operator (Aghdam and Davison 2004). It is to be noted that in the case of discrete-time control, this performance index takes intersample ripple effects into account.

The following cases are examined: 
i) Optimal continuous-time decentralized LTI controller: Consider applying the continuous-time controller:

$$
u(t)=\operatorname{diag}\left(k_{1}, k_{2}\right) y(t)
$$

to (14). Then, on minimizing the performance index defined in (15) with respect to $k_{1}$ and $k_{2}$, the optimal gains $k_{1}=3.332, k_{2}=-6.577 \times 10^{2}$, and the performance index of $J_{o p}=1.105 \times 10^{5}$ are obtained. The resultant performance index for $x(0)=\left[\begin{array}{lll}1 & 1 & 1\end{array}\right]^{\prime}$ is $1.087 \times 10^{5}$ and Figure 5 gives the corresponding input and output signals for this case. Note that $y_{1}(0)=1$, $y_{2}(0)=-0.995$ and $y_{1}$ then peaks to a value of 32.95 before settling down to zero. The performance of the resultant controller is not satisfactory, and is a consequence of the large ADFM at 0.1 in the system. In fact, even using a decentralized continuous-time LTI dynamic controller will not make a significant improvement in the performance of the system in presence of the large ADFM, e.g., on minimizing the performance index (15) with respect to parameters of a first order dynamic controller for each control agent, an improvement of only $2.2 \%$ is achieved in the resulting optimal performance index $J_{o p}$.
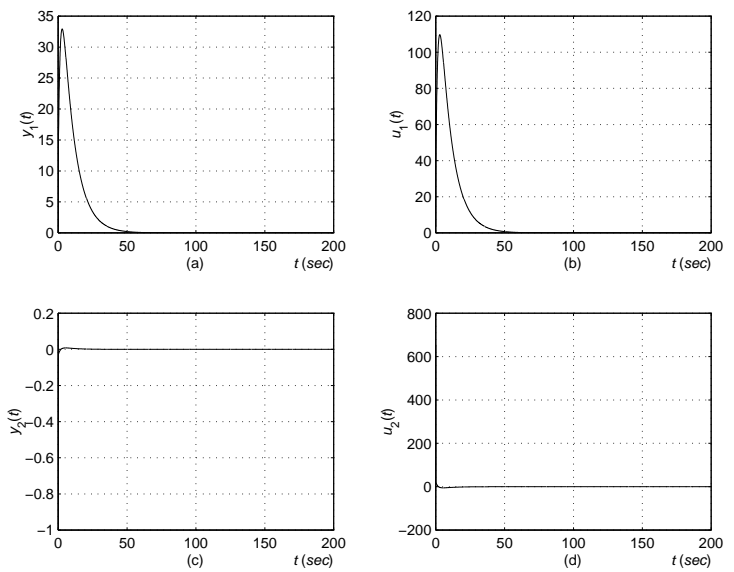

Fig. 5. Closed-loop simulation results for Example 1, using optimal decentralized continuous-time LTI controller. (a) Output signal of control agent 1 $\left(\max \left(\left|y_{1}(t)\right|\right)=32.95\right)$; (b) input signal of control agent 1 $\left(\max \left(\left|u_{1}(t)\right|\right)=109.8\right)$; (c) output signal of control agent 2 $\left(\max \left(\left|y_{2}(t)\right|\right)=8.122 \times 10^{-3}\right) ;(\mathrm{d})$ input signal of control agent $2\left(\max \left(\left|u_{2}(t)\right|\right)=654.5\right)$.

ii) Decentralized discrete-time controller: Consider now applying the discrete-time controller:

$$
u[k]=\operatorname{diag}\left(k_{1}, k_{2}\right) y[k]
$$

to (14), with a sampling period of $T>0$ and a $\mathrm{ZOH}$. Then on minimizing the performance index (15) as was done in (i), using Algorithm 1 in (Aghdam and Davison 2004), with respect to $k_{1}, k_{2}$ and $T$, the optimal performance index of $J_{o p}=4896$ is obtained which is approximately 20 times smaller than the result obtained in (i). The corresponding optimal gains are given by $k_{1}=9.996$ and $k_{2}=-7.069 \times 10^{-1}$, and the optimal sampling period is $T_{o p}=3.085 \mathrm{sec}$; the performance index obtained for $x(0)=\left[\begin{array}{lll}1 & 1 & 1\end{array}\right]^{\prime}$ is $2.116 \times 10^{4}$ and Figure 6 gives the resultant input and output signals for this case. The transient response of this system now has a much more reasonable magnitude compared to Figure 5.
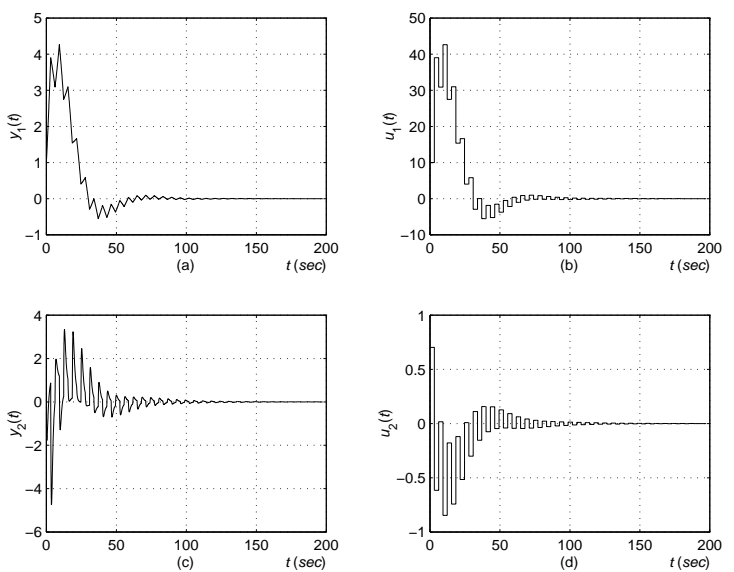

Fig. 6. Closed-loop simulation results for Example 1, using optimal decentralized discrete-time controller. (a) Output signal of control agent 1; (b) input signal of control agent 1; (c) output signal of control agent 2; (d) input signal of control agent 2 .

iii) GSHFs in the form of first-order polynomials: Consider now applying sampled-data hold functions $f_{1}(t)=a_{1} t+b_{1}$ and $f_{2}(t)=a_{2} t+b_{2}$ to each control agent. In this case, a two phase process is used: first, on using the result of Lemma 1 , the coefficients $a_{1}$, $b_{1}, a_{2}$ and $b_{2}$ are obtained in such a way, that the magnitude of the unstable ADFM in terms of minimum condition measures in the discrete-time domain (see Definition 5) is minimized. Then on tuning the GSHF coefficients obtained, to minimize the performance index (15) using Algorithm 1 in (Aghdam and Davison 2004), the following results are obtained for the optimal sampled-data hold functions:

$$
\begin{aligned}
& \text { Optimal } f_{1}(t): f_{1}(t)=-4.886 t+2.915 \\
& \text { Optimal } f_{2}(t): f_{2}(t)=1.768 t-1.978
\end{aligned}
$$

where the optimal sampling period obtained is $T_{o p}=$ $1.152 \mathrm{sec}$. The resultant minimum performance index in this case is $J_{o p}=15.08$. Note that in this case only one parameter has been added to the control design of each agent, compared to discrete-time control with a $\mathrm{ZOH}$, but a significant improvement has been achieved. The corresponding input and output signals of control agent 1 and control agent 2 for $x(0)=\left[\begin{array}{lll}1 & 1 & 1\end{array}\right]^{\prime}$ are depicted in Figure 7 in which (a) and (b) give the output and input signals of control agent 1 , and (c) and (d) give the corresponding signals of control 
agent 2 , respectively. The resultant performance index for $x(0)=\left[\begin{array}{lll}1 & 1 & 1\end{array}\right]^{\prime}$ is 50.17, which is significantly smaller than the value obtained by using a $\mathrm{ZOH}$ in case (ii).
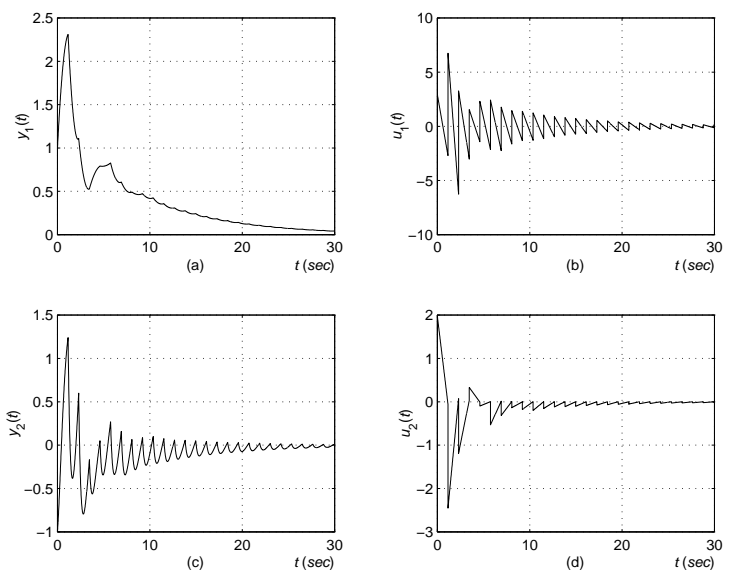

Fig. 7. Closed-loop simulation results for Example 1, using optimal decentralized discrete-time controller with first-order GSHFs (18a), (18b). (a) Output signal of control agent 1 ; (b) input signal of control agent 1; (c) output signal of control agent 2 ; (d) input signal of control agent 2 .

It is clear that the performance indices obtained for the optimal discrete-time decentralized controller of case (ii) and the optimal GSHFs of case (iii) are superior to the continuous-time decentralized controller (16). It is to be noted that to simplify the implementation problem, one can also use the GSHF design method given in (Aghdam 2006) to obtain a piecewise constant hold function instead of a polynomial one.

So far, various output feedback controllers have been considered in this example. It is to be noted for this problem, that the minimum achievable performance index for (15) using any type of controller is obtained by using the centralized continuous-time state feedback law, and the corresponding performance index for $x(0)=\left[\begin{array}{lll}1 & 1 & 1\end{array}\right]^{\prime}$ is given by $J_{o p}=1.448$.

Remark 6 It is to be noted that the optimization algorithms used for discrete-time controller design in Example 1, minimize the continuous-time quadratic performance index (15). This implies that the optimization algorithms take intersample ripple effects into account.

\section{Conclusions}

In this paper, a class of decentralized linear time-varying (LTV) controllers has been proposed for continuoustime linear time-invariant (LTI) plants, using discretetime control. The controllers obtained have the property that when the plant has a dominant mode which is an approximate decentralized fixed mode (ADFM) of large magnitude and an approximate quotient fixed mode (AQFM) of small magnitude, then discrete-time controls can outperform their continuous-time LTI counterparts. It should be noted, however, that in designing such discrete-time controllers, one cannot choose "fast" sampling intervals; this implies that the resulting control obtained, although "better" than any continuous-time control, cannot have high performance. This is the price to pay for having an ADFM. A condition under which repeated decentralized fixed modes (DFM) can be eliminated by using a continuous-time feedback followed by sampling has also been given. The performance of the closed-loop system depends on the type of hold function used in the discrete to continuous process, the sampling interval, and the control law itself. Optimization algorithms to design discrete-time controllers which account for intersample ripple are then described.

Simulation results obtained using an optimal sampling interval and optimal static output feedback control, show significant improvements compared to the corresponding continuous-time optimal decentralized controller. They also show that using generalized sampleddata hold functions (GSHF) instead of a simple $\mathrm{ZOH}$, in the proposed decentralized discrete-time controller, can introduce more degrees of freedom in the discrete-time controller design, which results in an improvement in the overall performance of the system.

\section{Acknowledgment}

The authors would like to thank Dr. J. L. Willems for his useful comments.

\section{References}

Aghdam, A. G. (2006). Decentralized control design using piecewise constant hold functions. In: Proceedings of the 2006 American Control Conference. pp. 207-212.

Aghdam, A. G. and E. J. Davison (1999). Decentralized control of systems, using generalized sampled-data hold functions. In: Proceedings of the 38'th IEEE Conference on Decision and Control. pp. 3912-3913.

Aghdam, A. G. and E. J. Davison (2004). An optimization algorithm for decentralized digital control of continuoustime systems which accounts for inter-sample ripple. In: Proceedings of the 2004 American Control Conference. pp. 4273-4278.

Aghdam, A. G., E. J. Davison and R. Becerril-Arreola (2006). Structural modification of systems using discretization and generalized sampled-data hold functions. Automatica 42, 1935-1941.

Anderson, B. D. O. and D. J. Clements (1981). Algebraic characterization of fixed modes in decentralized control. Automatica 17, 703-712.

Anderson, B. O. D. and J. B. Moore (1981). Time-varying feedback laws for decentralized control. IEEE Transactions on Automatic Control AC-26(5), 1133-1138.

Chammas, A. B. and C. T. Leondes (1978). On the design of linear time invariant systems by periodic output feedback, part i and ii. International Journal of Control 27, 885-903. 
Davison, E. J. and T. N. Chang (1990). Decentralized stabilization and pole assignment for general proper systems. IEEE Transactions on Automatic Control AC-35(6), 652-664.

Feuer, A. and G. C. Goodwin (1994). Generalized sample hold functions-frequency domain analysis of robustness, sensitivity, and intersample difficulties. IEEE Transactions on Automatic Control AC-39(5), 1042-1047.

Gong, Z. and M. Aldeen (1997). Stabilization of decentralized control systems. Mathematical Systems, Estimation, and Control 7(1), 1-16.

Kabamba, Pierre T. (1987). Control of linear systems using generalized sampled-data hold functions. IEEE Transactions on Automatic Control AC-32(9), 772-783.

Khargonekar, P. P. and A. B. Özgüler (1994). Decentralized control and periodic feedback. IEEE Transactions on Automatic Control 39(4), 877-882.

Kobayashi, H. and T. Yoshikawa (1982). Graph-theoretic approach to controllability and localizability of decentralized control systems. IEEE Transactions on Automatic Control AC-27(5), 1096-1108.

Lavaei, J. and A. G. Aghdam (2007a). A graph theoretic method to find decentralized fixed modes of LTI systems. Automatica, to appear.

Lavaei, J. and A. G. Aghdam (2007b). Optimal periodic feedback design for continuous-time LTI systems with constrained control structure. International Journal of Control 80(2), 220-230.

Lavaei, J. and A. G. Aghdam (2007c). Simultaneous LQ control of a set of LTI systems using constrained generalized sampleddata hold functions. Automatica 43, 274-280.

Özgüner, U. and E. J. Davison (1985). Sampling and decentralized fixed modes. In: Proceedings of the 1985 American Control Conference. pp. 257-262.

Scattolini, R. and N. Schiavoni (1996). Decentrlaized control of multirate systems subject to arbitrary exogenous signals. IEEE Transactions on Automatic Control 41(10), 15401544 .

Sezer, M. E. and D. D. Šiljak (1981). Structurally fixed modes. Systems and Control Letters 1(1), 60-64.

Vaz, A. F. and E. J. Davison (1989). On the quantitative characterization of approximate decentralized fixed modes using transmission zeros. Mathematics of Control Signals, and Systems 2, 287-302.

Wang, S. H. and E. J. Davison (1973). On the stabilization of decentralized control systems. IEEE Transactions on Automatic Control AC-18, 473-478.

Willems, J. L. (1988). Elimination of fixed modes in decentralized systems by means of sampling. Systems and Control Letters 10, 1-8. 